\title{
Active Network Management via Agent Technology
}

\author{
Yasin Kaplankiran, Alexander Keiblinger, Hermann Többen \\ Institute for Systems Analysis and IDP, TU-Berlin/DAI-Lab \\ Franklinstraße 28/29, 10587 Berlin, Germany \\ \{kaplan, keiblin, toebben\}@Cs.tu-berlin.de
}

Key words: Intelligent Agents, VPNs, active networks, IP-tunneling, marketplaces

\begin{abstract}
Active networks represent a significant step in the evolution of packetswitched networks, from traditional packet-forwarding engines to more general functionality supporting dynamic control and modification of network behavior. This paper describes a basic framework for an active network management system based upon agent technology, which can provide different views on a network, each related to a specific problem domain. Exemplarily the domain of VPN provisioning will be presented by a VPN management service covering all kind of aspects needed to provide VPNs for a wide quantity and variety of customers.
\end{abstract}

\section{INTRODUCTION}

Telecommunication providers change from voice carriers to suppliers of differentiated services. They need to leverage their existing network infrastructure to manage it more efficiently and enhance it for service provisioning of today's and future services. Active Networking is a promising new technology, delivering a distributed infrastructure for service provisioning. Active Networks promise to fulfil both requirements, the efficient and flexible management of network elements and networks as well as the fast introduction and provision of new services.

This paper describes an agent based Active Networking architecture. In addition we use the JIAC (Java based Intelligent Agent Componentware)

The original version of this chapter was revised: The copyright line was incorrect. This has been corrected. The Erratum to this chapter is available at DOI: 10.1007/978-0-387-35522-1_37 
agent platform, which has been developed at the DAI-Labor. JIAC is an open and scalable agent architecture. Intelligent agents are pieces of software which act autonomously on behalf of a user or another agent. Agents have the ability to find and filter information, negotiate for services, easily automate complex tasks or collaborate with other software agents to solve complex problems. Mobile agents can even migrate between network nodes to accomplish a task nearby the necessary resources.

We use the setup and administration of VPN's respective their virtual links as a scenario for agent based active networking in this paper due to the overlap of VPN management needs and agent technology promises.

While the demand for VPNs grows rapidly, the supply lacks. VPNs are still very hard to set up and to maintain. To be able to satisfy the future demand, it will be necessary to greatly reduce the effort for VPN provision demand and customizable network services. The need for detailed control down to the single network element has led to the concept of Active Networks. This trend is further supported by ever falling costs for computing power, enabling the provision of mightful computing resources on network elements [3].

Active Networks remove the distinction between the network and connected computing devices by enabling the dynamic execution of program code on the network elements themselves. With the network becoming programmable, users can dynamically customize the network resources to their needs. Hence the dynamic programing of network nodes enables network as well as service providers to integrate new services into their networks easily and quickly and to manage their network efficiently [1].

\section{ACTIVE NETWORKING BASED ON AOT}

Agent technology is a new approach of designing software. Agents are active software components, which can either reside at specific locations in the network (stationary agents) or travel between network nodes (mobile agents). Agents have the ability to find and filter information, negotiate for services, automate complex tasks and collaborate with other software agents to solve even more complex problems.

The agent oriented technology (AOT) is well suited for network management [2]. It can be effectively used to decentralize network management activities [5]. In particular the characteristic mobility, co-operation and 
autonomy of the agents emphasize the advantages of AOT in distributed management applications. AOT is an approach to fulfill the prerequisites of active nodes and mobile program code in active networking.

\section{JIAC - JAVA BASED AGENT ARCHITECURE}

An agent based solution confirming to the structure of JIAC consists of Agents and marketplaces as the two elementary components. According to their ability to migrate agents can be further divided into two basic flavors: stationary and mobile agents. Marketplaces as the basic agent platform provide the environment in which stationary agents reside, offering their services to other stationary or mobile agents visiting the marketplace. JIAC marketplaces need a JAVA execution environment, namely a Java Virtual Machine (JVM). Every marketplace contains a manager agent, being responsible for administering the marketplace and providing basic administrative services to the other agents on the marketplace.

The stationary agents reside on a certain marketplace, which means they are constantly assigned to that marketplace. The following types of agents may be considered stationary agents: the marketplace manager agents, service provider agents and content provider agents. Mobile agents have the capability to travel from one marketplace to other marketplaces in an effort to find a suitable service provider agent. Mobility is one of the features supported by the use of marketplaces. It enables an agent to travel across the network to a marketplace where the resources it intends to use are locally accessible, thus reducing network load and communication latency (see also [4]). Given the ability of the active network components as described above, we envision the following management architecture (c.f. 1). 


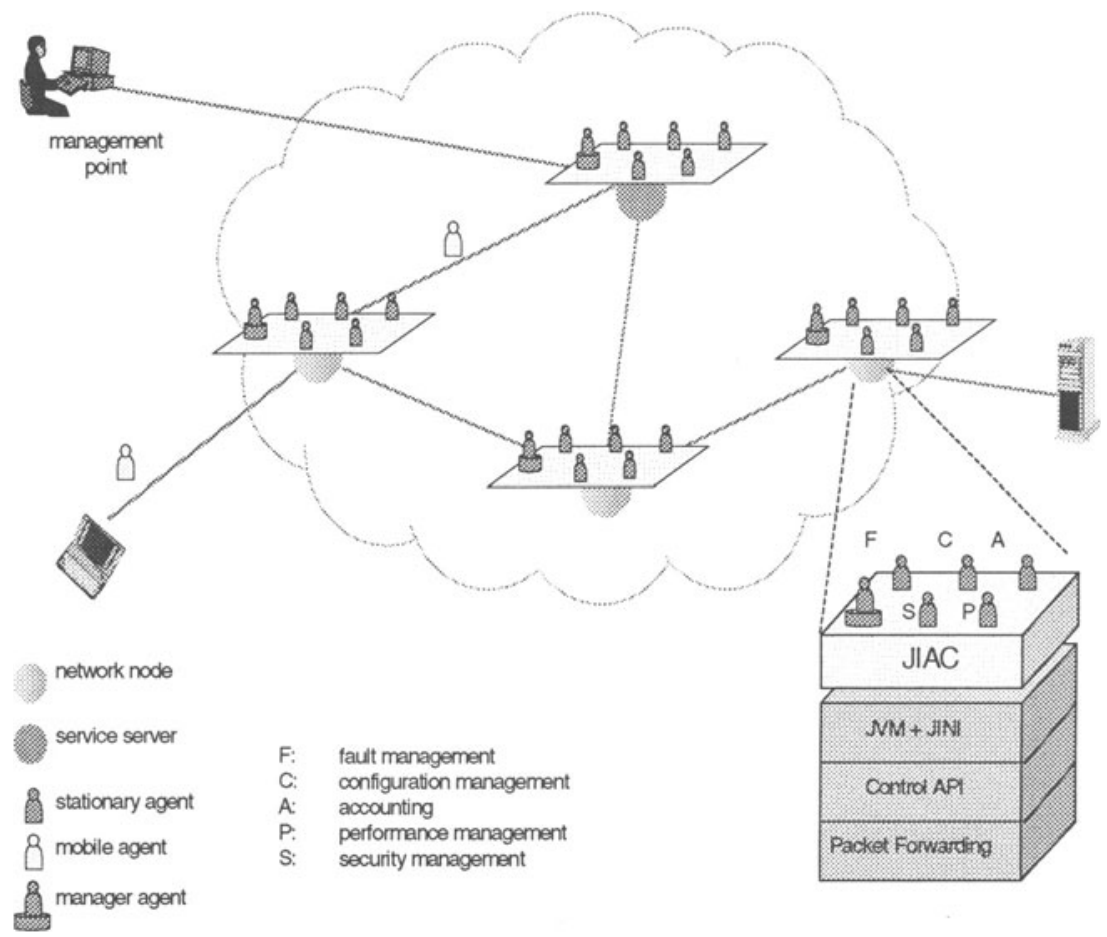

Fig. 1 Agent-based network management

On every active network node there will be established an agent platform. The agent platform consists of a marketplace (the yellow box labeled JIAC) with a manager agent and some stationary agents, providing standard functionality and mobile agents as well. Naturally the agents can communicate with each other via the network itself. Migration as a process is embedded with the communication. The stationary agents provide the basic network management functions known as FCAPS in the OSI-Management area. The brokering between agents' services and legacy systems can be provided via a JINI environment [4].

\subsection{Capabilities of JIAC-Agents}

The JIAC agent architecture provides the designer of agent systems with a broad range of distinct capabilities. On one hand agents are able to communicate and to cooperate with each other on the base of speech-acts and interaction protocols. Within their activities JIAC agents make up their deci- 
sions upon knowledge, which is represented in an adequate language called JIAC Agent Description Language (JADL). Moreover the agents are equipped with sophisticated planing capabilities. The latter gives benefit towards the configuration problems concerning the setup of connections within a heterogeneous network.

\section{VPN MANAGEMENT SERVICE}

In this chapter we will present Virtual Private Networking (VPNs) as a possible „killer application“ for agent based Active Networking. To achieve this goal, we will present an agent based design for the dynamic creation as well as the management of VPN's.

We understand a VPN as a private network, connecting remote locations using public networking infrastructure like the Internet. Using packet tunneling abstracts from the underlying network infrastructure and guarantees data security with encryption mechanisms.

\subsection{Motivation}

We regarded VPN provisioning from a global point of view to identify the main aspects. Regarding not only the operational phase of VPNs, but the complete process from planning to deployment, including maintenance and expansion of the VPN. 


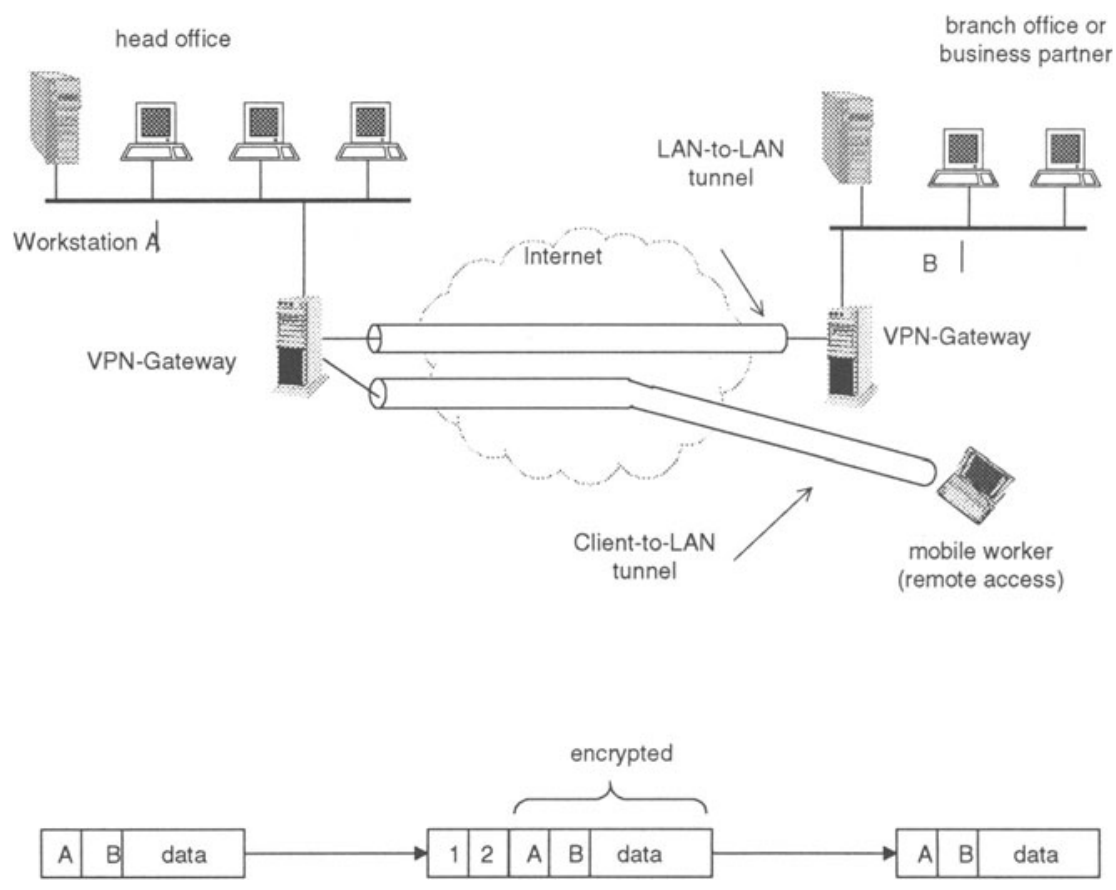

Fig. 2: Example of a VPN scenario

Figure 2 shows a usual VPN scenario. There is a virtual corporate network connecting a headquarter with branches and business partners using secure VPN lines. In addition, there is a remote workforce connected to the Intranet business data of the company using remote access software also using VPN technology.

\subsection{The business model}

Life-cycle of a corporate VPN installation. Business model characterizes the main four phases of this process: the specification (Agents $S$ ) of the customers demand reflected by the Service Level Agreements (SLAs), the configuration of the involved network-components (Agents $\mathrm{C}$ ), the accounting and billing (Agents A), the testing and finally the operational use (Agent F, c.f. 3). 


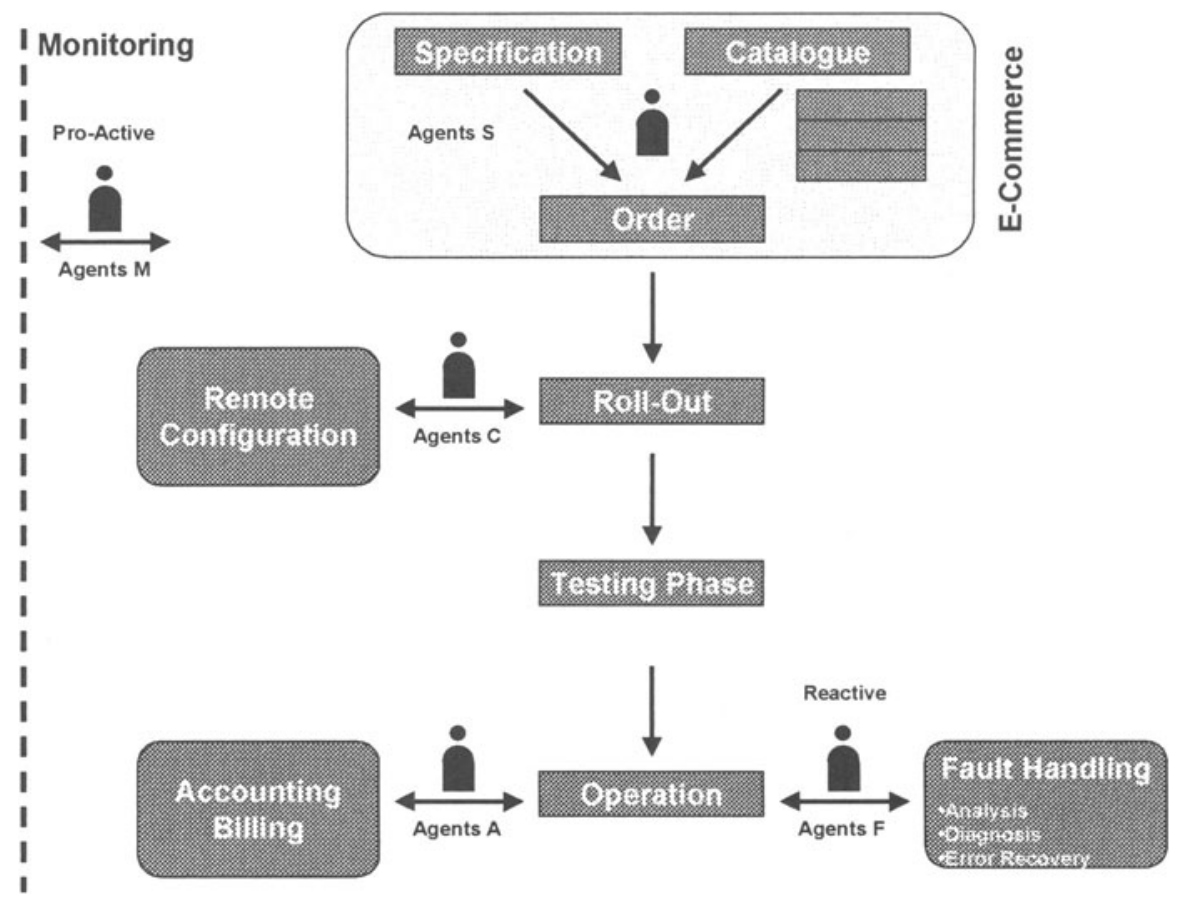

Fig. 3: VC-Market

Due to the above defined phases we have identified five areas where agents could be applied within this process:

- Specification Agents (area S):

Agents are used to help in the specification of a new VPN. This is done by taking customer requirements and existing networking hardware into account and mapping those onto predefined VPN templates of the provider. This process is iterative and relies expert knowledge from a database. $\mathrm{Hu}$ man experts are consulted if necessary. As an example you could imagine an agent based e-commerce environment, using a web front end as an automated customer point of contact and a call-center staffed with experts for customer advice in the background.

- Configuration Agents (area C):

Agents will be used in the roll-out phase, when newly installed VPN hardware must be configured to the specific needs of the customer. Remote configuration agents will take care of configuration issues, taking the burden 
off the shoulders of the installing technicians or customer staff. When the VPN has passed the test phase and is in production use, the next two areas in which we would employ agent technology are:

- Fault-Management Agents (area F):

Agents of this kind would be triggered when a problem occurs. They analyze the problem, diagnose the cause and attempt to solve it. These agents would be equipped with components to cope with many of the standard problems. They contact specialized agents or as a last option human network managers for unknown problems and unusual error conditions, which they are unable to resolve.

- Accounting and billing agents (area A):

Agents are responsible for accounting and billing. They collect accounting information in their decentral location and map the accounting data on billing items. In addition to the standard accounting and billing components, providers can add their own agents to facilitate special accounting schemes and billing rules.

- Monitoring agents (area M):

In parallel to all these process steps, monitor agents will check for conformance to the specification, errors or performance problems. The monitor agents provide data which will be used by other agents or they spawn the fault management agents described above on detection of errors.

\section{CONCLUSIONS}

Agent oriented Technology seems to be well suited for network management. It can be effectively used to decentralize network management activities. In this paper the described an agent based framework for active networking. Within this approach every active network node will be equiped with an agent marketplace encompassing stationary agents which provide basic OSI-management functionalities and a manager agent for administrating the marketplace itself. As a possible „killer application“ for agent based Active Networking we described the dynamic configuration of an VPN. Telcos will benefit directly from the results of the research and the possible deployment of the developed technology in their networks. Agent based network management can help to reduce their network management costs, increase flexibility and offer new business opportunities by enabling new types of services. 


\section{REFERENCES}

[1] Sahin Albayrak: "Intelligent Agents for Telecommunications Applications“, IOS Press, June 1998

[2] Sahin Albayrak, Francisco J. Garijo (Eds.), ,Intelligent Agents for Telecommunications Applications“, Second International Workshop, IATA'98, Springer, July 1998

[3] David L. Tennenhouse, et.al.: "A Survey of Active Network Research“, IEEE Communications Magazine, pp.80-85, Vol. 35, No. 1, January 1997

[4] Sun® Microsystems: "JiniTM Technology“, http://www.sun.com/jini/

[5] R. Weihmayer, R. Brandau: ,A Distributed AI Architecture for Customer Network Control“" in ,IEEE“, pp. 656-662, 1990 